\title{
Erratum to: Multiple Scattering Theory for Spectroscopies
}

Didier Sébilleau, Keisuke Hatada and Hubert Ebert

Erratum to:

D. Sébilleau et al. (eds.), Multiple Scattering Theory

for Spectroscopies, Springer Proceedings in Physics 204, https://doi.org/10.1007/978-3-319-73811-6

In the original version of the book, belated correction from author to update the frontmatter with revised acknowledgement content has to be incorporated. The erratum book has been updated with the change. 